EPJ Web of Conferences 43, 03008 (2013)

DOI: $10.1051 /$ epjconf/20134303008

(C) Owned by the authors, published by EDP Sciences, 2013

\title{
Amplitudes of solar-like oscillations in red giants: Departures from the quasi-adiabatic approximation
}

\author{
R. Samadi ${ }^{1, a}$, K. Belkacem¹, M.-A. Dupret ${ }^{2}$, M.J. Goupil ${ }^{1}$, H.-G. Ludwig ${ }^{3}$, \\ C. Barban ${ }^{1}$, F. Baudin ${ }^{4}$ and E. Caffau ${ }^{3,5}$
}

${ }^{1}$ LESIA, CNRS, Université Pierre et Marie Curie, Université Denis Diderot, Observatoire de Paris, 92195 Meudon Cedex, France

${ }^{2}$ Institut d'Astrophysique et de Géophysique de l'Université de Liège, Allée du 6 Août, 17, 4000 Liège, Belgium

${ }^{3}$ Zentrum für Astronomie der Universität Heidelberg, Landessternwarte, Kïnigstuhl 12, 69117 Heidelberg, Germany

${ }^{4}$ Institut d'Astrophysique Spatiale, CNRS, Université Paris XI, 91405 Orsay Cedex, France

${ }^{5}$ GEPI, CNRS, Observatoire de Paris, Université Denis Diderot, Place Jules Janssen, 92195 Meudon Cedex, France

\begin{abstract}
CoRoT and Kepler measurements reveal us that the amplitudes of solar-like oscillations detected in red giant stars scale from stars to stars in a characteristic way. This observed scaling relation is not yet fully understood but constitutes potentially a powerful diagnostic about mode physics.

Quasi-adiabatic theoretical scaling relations in terms of mode amplitudes result in systematic and large differences with the measurements performed for red giant stars. The use of a non-adiabatic intensityvelocity relation derived from a non-adiabatic pulsation code significantly reduces the discrepancy with the CoRoT measurements. The origin of the remaining difference is still unknown. Departure from adiabatic eigenfunction is a very likely explanation that is investigated in the present work using a 3D hydrodynamical model of the surface layers of a representative red giant star.
\end{abstract}

\section{INTRODUCTION}

Before CoRoT (launched in December 2006), solar-like oscillations had been detected for a dozen of bright red giant stars, mostly from the ground, i.e. in terms of Doppler surface velocity. These early observations already show us that the maximum of the mode surface velocity (hereafter $V_{\max }$ ) scale from star to star in a characteristic way. Comparaison between observed and modelled scaling relations in terms of mode amplitudes potentially provides powerful diagnostics about turbulent convection, mode physics and structure of the stellar surface $[1,2]$.

On the basis of the theoretical calculations of [3] done for main sequence stars and subgiants, [4] have derived the first example of a scaling law for $V_{\max }$. This scaling law predicts that $V_{\max }$ varies as $(L / M)^{s}$ with a slope $s \simeq 1, L$ the luminosity, and $M$ the mass of the star. The theoretical calculations of [3] were based on the assumption that there is an equipartition between the energy carried by the most energetic eddies and the modes (for a discussion about this assumption see [5] and [1]). Following the scaling proposed by [4], other theoretical scaling laws have been proposed that results in different values of the slope $s[6,7]$.

\footnotetext{
ae-mail: reza.samadi@obspm.fr
}

This is an Open Access article distributed under the terms of the Creative Commons Attribution License 2.0, which permits unrestricted use, distribution, and reproduction in any medium, provided the original work is properly cited. 
Ground-based Doppler velocity measurements done for main-sequence stars favour a slope close to $s=0.7$ in agreement with [7] theoretical scaling relation. [8] have recently extended the calculations performed by [7] for main-sequence stars to sub- and red giant stars. Their calculations, which are presented in Sect. 2, results in underestimation by $30 \%$ of the measurements made from the ground in terms of Doppler velocity for red giant stars. However, it is worthwhile to note that the number of Doppler velocity measurements available for red giants are too small to firmly conclude.

Since the launches of the CoRoT and Kepler space-crafts, solar-like oscillations have now been detected on several thousands red giant stars. Due to their high amplitudes, these modes can been accurately measured in terms of frequency, amplitudes and lifetimes. These measurements are, however, all made in terms of intensity and depend on the instrument bandpass. It is first required to convert amplitudes measured in the instrument bandpass into bolometric ones [9, 10]. In order to compare predicted with measured mode amplitudes, it is next necessary to relate mode-velocity amplitudes to bolometric ones. The easy way to connect velocity amplitude is to assume quasi-adiabatic oscillations (and an isothermal atmosphere). This yields a simple relation between the maximum of the bolometric amplitude, $(\delta L / L)_{\max }$, and $V_{\max }$ (see [4]). However, as shown by [8], the use of such relation together with the current theoretical predictions in terms of velocity amplitude results in an under-estimation by a factor about 2.5 of the bolometric mode amplitudes measured by [11] on a set of CoRoT red giant stars. [8] have investigated the impact of using a non-adiabatic intensity-velocity relation. They have established such non-adiabatic relation with the MAD non-adiabatic pulsation code [12]. This nonadiabatic relation significantly reduces the discrepancy with the CoRoT data. This result is summarised in Sect. 3.

Nevertheless, the current theoretical amplitudes remain $40 \%$ below the CoRoT measurements. Since the (few) velocity measurements available for red giants are also underestimated, this suggests that we still under-estimate the mode driving for those stars. As suggested by [8], a possible reason of this under-estimation could come from the use of adiabatic eigenfunction for calculating the mode driving.

Calculations of mode driving made up to now for the case of the Sun [13-17] and other stars $[6,8,18-20]$ were undertaken using either adiabatic eigenfunctions [8, 17, 19, 20] or nonadiabatic eigen-functions $[6,13,14,16,21,22]$. Calculations using non-adiabatic eigenfunctions have only be performed with [23] pulsation code. However, theoretical mode amplitudes computed with these non-adiabatic eigenfunctions do not differ significantly from those computed with adiabatic eigenfunctions [6]. Furthermore, non-adiabatic eigenfunctions computed with the MAD code [12] differ with the adiabatic ones only in a small fraction of the excitation region. However, both non-pulsation codes are based on time-dependent versions of the mixing-length theory, which is well known to be a crude formulation of convection. Therefore a more realistic and consistent non-adiabatic approach that does not rely on free parameters and that includes constraints from 3D hydrodynamical models is required. Toward this goal, we investigate in the present work (Sect. 4) the departure from adiabatic eigenfunction using constraints obtained from two 3D hydrodynamical models of the surface layers of stars.

\section{MODE AMPLITUDE IN VELOCITY}

The root mean-square surface velocity, $v_{\text {rms }}$, of a given radial mode is related to the mode excitation rate (or energy supply rate), $\mathcal{P}$, and the mode lifetime, $\tau$, as [1]

$$
v_{\mathrm{rms}}^{2}(v)=\frac{\tau}{2} \frac{\mathcal{P}}{\mathcal{M}}
$$

with

$$
\mathcal{M}(v) \equiv \frac{1}{\left|\xi_{\mathrm{r}}\right|^{2}\left(r_{s}\right)} \int_{0}^{M}\left|\xi_{\mathrm{r}}\right|^{2} \mathrm{~d} m,
$$




\section{Ageing Low Mass Stars: From Red Giants to White Dwarfs}

where $v$ is the mode frequency, $M$ the mass of the star, $\xi_{\mathrm{r}}$ the radial mode eigen-displacement, and $r_{s}$ the layer in the atmosphere where the mode velocity is supposed to be measured.

Different formalisms have been proposed for evaluating the energy supply rate $\mathcal{P}$ [14-16, 21, 24-27]. All of them are inspired by the pionner model by [28]. They, however, differ from each other either by the way turbulent convection is described, or by the adopted sources of driving. Among the sources of driving, two major mechanisms are usually considered: the mechanical driving due to the Reynolds stresses, and a thermal source of driving, which arise from the advection of the entropy fluctuations by the turbulent velocity field [26].

In the formalism by [26], $\mathcal{P}$ has the following expression

$$
\mathcal{P}=\frac{1}{8}\left(C_{R}^{2}+C_{S}^{2}\right)
$$

where $C_{R}^{2}$ and $C_{S}^{2}$ the contribution of the Reynolds stress and the entropy fluctuations, respectively. The expressions for $C_{R}^{2}$ and $C_{S}^{2}$ are

$$
\begin{aligned}
& C_{R}^{2} \propto \int_{0}^{M} \rho_{0}\left(\frac{d \xi_{\mathrm{r}}}{d r}\right)^{2} S_{R}\left(r, \omega_{0}\right) \mathrm{d} m \\
& C_{S}^{2} \propto \int_{0}^{M} \frac{\alpha_{s}^{2}}{\rho_{0}} g_{r} S_{S}\left(r, \omega_{0}\right) \mathrm{d} m,
\end{aligned}
$$

where $\alpha_{s}=\left(\partial P_{g} / \partial s\right)_{\rho}, P_{g}$ is the gas pressure, $s$ the entropy, $\rho_{0}$ the density, $S_{R}$ and $S_{S}$ are the "source terms" associated with the Reynolds stresses and entropy fluctuations respectively, and finally $g_{r}\left(\xi_{\mathrm{r}}, r\right)$ a function that involves the first and second derivatives of $\xi_{\mathrm{r}}[26]$. The source functions, $S_{R}$ and $S_{S}$, involve the dynamic properties of the turbulent medium. Their importance for the driving are not discussed in this paper (for a review see [1]).

[8] have computed $\mathcal{P}$ and $\mathcal{M}$ for a set of sub- and red giant 3D models. They have found that the maximum of the ratio $(\mathcal{P} / M)$ (see Eq. (1)) scales as

$$
\left(\frac{\mathcal{P}}{\mathcal{M}}\right)_{\max } \propto\left(\frac{L}{L_{\odot}} \frac{M_{\odot}}{M}\right)^{s}\left(\frac{M}{M_{\odot}} \frac{R_{\odot}^{3}}{R^{3}}\right)^{p / 2},
$$

where $L$ is the luminosity, $R$ the radius, $M$ the mass, $s=2.6$, and $p$ a coefficient that depends on the layer in the atmosphere where the velocity is evaluated (or measured). From now on, quantities with the subscript $\odot$ will refer to solar values. We have $p=2.0$ at the photosphere, and $p=2.1$ at the layer where spectrographs dedicated to stellar seismology are believed to be the most sensitive [19]. According to Eq. (1) and Eq. (6), the maximum of the mode velocity scales then as

$$
V_{\text {max }}=v_{0} \sqrt{\frac{\tau_{\max }}{\tau_{0}}\left(\frac{L}{L_{\odot}} \frac{M_{\odot}}{M}\right)^{s}\left(\frac{M}{M_{\odot}} \frac{R_{\odot}^{3}}{R^{3}}\right)^{p / 2}},
$$

where $\tau_{\max }$ is the mode lifetime (in days) at the peak frequency $\left(v_{\max }\right), \tau_{0}=3.88$ days and $v_{0}=0.31 \mathrm{~m} / \mathrm{s}$, when the velocity is evaluated at the photosphere, and $v_{0}=0.41 \mathrm{~m} / \mathrm{s}$ when it is evaluated at the layer appropriated for a comparison with Doppler velocity measurements.

The calculation of $V_{\max }$ (Eq. (7)) requires the mode lifetime at the peak frequency, $\tau_{\max }$. We consider the seismic measured of $\tau_{\max }$ done by [11] for about 170 CoRoT red giant targets. The scaling relation given by Eq. (7) is compared with the stars whose $V_{\max }$ has been measured so far in Doppler velocity from the ground. Accordingly, we computed $V_{\max }$ with $p=2.1$ and $v_{0}=0.41 \mathrm{~m} / \mathrm{s}$, the result is shown and compared with the measurements in Fig. 1 (left panel). 

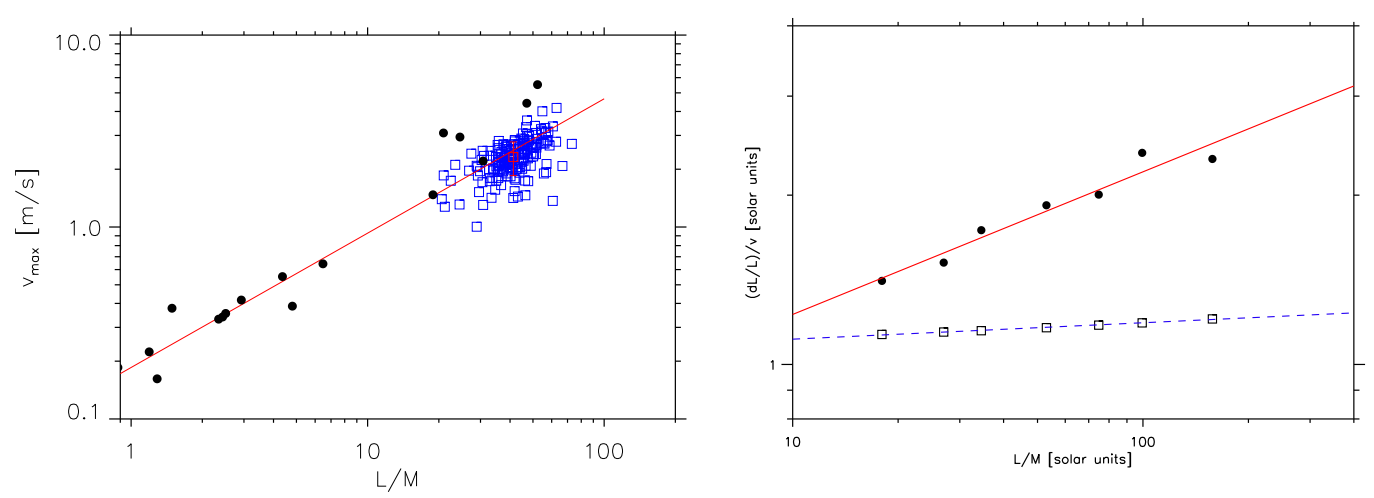

Figure 1. Left: Maximum of the mode velocity $V_{\max }$ as a function of $L / M$ [in solar units]. The filled circles correspond to the stars observed in Doppler velocity from the ground and the solid line to the power law of the form $(L / M)^{0.7}$ obtained by [7] using 3D models of main-sequence stars. The squares correspond to the theoretical $V_{\max }$ derived according to Eq. (7) (see Sect. 2). Right: Coefficient $\zeta$ (see Eq. 8) as a function of $L / M$ for sub- and red giants. The filled circles correspond to the values, $\zeta_{\text {nad }}$, obtained with the MAD non-adiabatic pulsation code (see details in the text). The empty squares correspond to the adiabatic coefficient (Eq. (9)). The solid line corresponds to a power law of the form $(L / M)^{k}$ with $k=0.25$. Both intensity-velocity relations $\zeta$ have been calibrated so that for the $\operatorname{Sun} \zeta=1$.

The theoretical values of $V_{\max }$ obtained for our subset of red giants are found to be close to the measurements obtained for the red giant stars observed so far in Doppler velocity from the ground. Note that the considerable dispersion seen in the theoretical values of $V_{\max }$ comes from the dispersion in the measured value of $\tau_{\max }$. As seen in Fig. 1, the theoretical $V_{\max }$ are found, on average, to be about $30 \%$ lower than the (few) measurements available for red giants.

\section{INTENSITY-VELOCITY RELATION}

We consider in this section quasi-adiabatic and non-adiabatic relations between $(\delta L / L)_{\max }$ and $V_{\max }$ and quantify their impact on mode amplitudes in terms of intensity. For convenience, [8] introduced the dimensionless coefficient $\zeta$ defined according to

$$
(\delta L / L)_{\max }=\zeta\left(\frac{\delta L}{L}\right)_{\odot}\left(\frac{V_{\max }}{v_{\odot}}\right),
$$

where $\left(\frac{\delta L}{L}\right)_{\odot}=2.53 \pm 0.11 \mathrm{ppm}$ is the maximum of the solar bolometric mode amplitude [9], and $v_{\odot}=18.5 \pm 1.5 \mathrm{~cm} / \mathrm{s}$ the maximum of the solar mode (intrinsic) surface velocity evaluated at the photosphere as explained in [20]. Note that the quantity $\zeta$ in Eq. (8) is defined at an arbitrary layer, which is conveniently choose at the photosphere (i.e. at $r=R_{*}$ ).

Assuming (quasi)-adiabatic modes and an isothermal yields the adiabatic coefficient $\zeta$ [4]

$$
\zeta_{\mathrm{ad}} \equiv \sqrt{\frac{T_{\mathrm{eff}, \odot}}{T_{\mathrm{eff}}}} .
$$

We computed $(\delta L / L)_{\max }$ according to Eq. (8) using the scaling law given by Eq. (7) for $V_{\max }$ (with $p=2.0$ and $v_{0}=0.31 \mathrm{~m} / \mathrm{s}$ ), and adopting the adiabatic coefficient $\zeta_{\text {ad }}$ (Eq. (9)). Figure 2 (left) shows $(\delta L / L)_{\max }$ as a function of ratio $(L / M)$. We also plotted the mode amplitudes measured for a small sample of CoRoT main-sequence stars (see [11] and references therein). Theoretical $(\delta L / L)_{\max }$ underestimates the amplitudes measured on the CoRoT red giant stars by a factor of about 2.5 . 


\section{Ageing Low Mass Stars: From Red Giants to White Dwarfs}
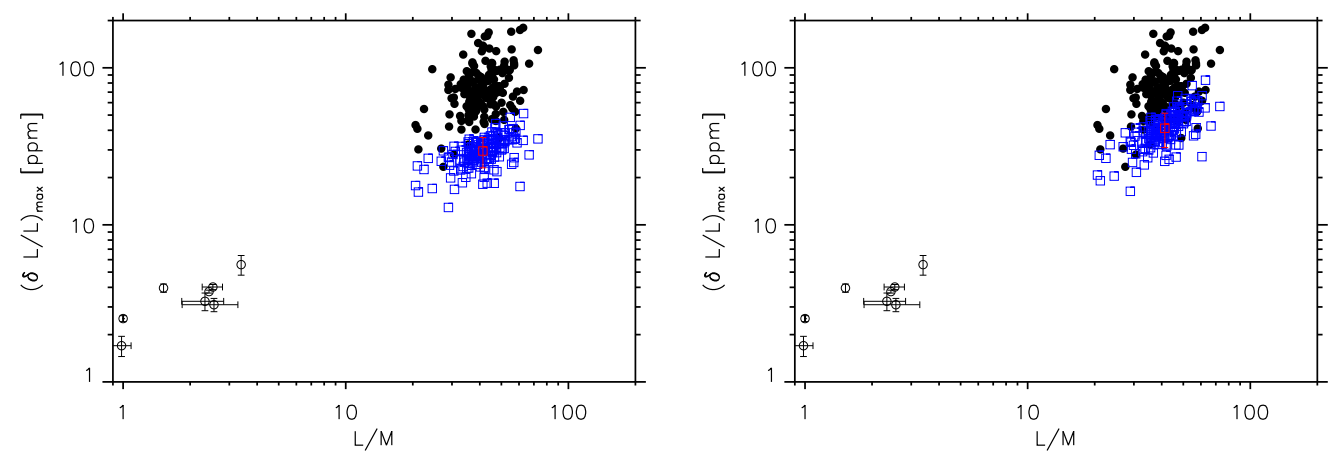

Figure 2. Left: Maximum of the mode intensity fluctuation $(\delta L / L)_{\max }$ as a function of $L / M$ [in solar units]. The filled circles correspond to the seismic measurements performed by [11] on a large number of CoRoT red giant stars ( $\sim 170$ targets). We only considered the targets for which the mode line width is broader than twice the frequency resolution. The empty circles correspond to the main-sequence stars observed so far by CoRoT (see [11]), and the squares are the theoretical $(\delta L / L)_{\max }$ computed according to the [4] adiabatic coefficient (Eq. (9), see text). Right: Same as left panel, the theoretical $(\delta L / L)_{\max }$ are computed here assuming for $\zeta$ the non-adiabatic scaling relation given by Eq. (10) (see also Fig. 1, right).

[8] have computed $\zeta$ using the MAD non-adiabatic pulsation code [12]. This code includes the time-dependent convection (TDC) treatment described in [12]. For sub- and red giant stars $(L / M \gtrsim$ $\left.10 L_{\odot} / M_{\odot}\right)$, the non-adiabatic intensity-velocity relation obtained with the MAD code was found to scale as

$$
\zeta_{\text {nad }}=\zeta_{0}\left(\frac{L}{L_{\odot}} \frac{M_{\odot}}{M}\right)^{k}
$$

where $k=0.25 \pm 0.05$ and $\zeta_{0}=0.59 \pm 0.07$. This result is illustrated in Fig. 1 (right) and compared with the adiabatic coefficient $\zeta_{\mathrm{ad}}$ (Eq. (9)). The non-adiabatic coefficient increases rapidly with increasing $(L / M)$ while $\zeta_{\text {ad }}$ remains almost constant. Hence, the higher $(L / M)$, the larger the difference between the non-adiabatic coefficient, $\zeta_{\text {nad }}$, and the adiabatic one, $\zeta_{\text {ad }}$.

We computed $(\delta L / L)_{\max }$ according to Eq. (8) assuming this turn the non-adiabatic scaling law given by Eq. (10). The result is shown in Fig. 2 (right). Using the non-adiabatic coefficient results in an increase of the bolometric amplitude by a factor $\sim 1.5$ compared to the calculations based on the adiabatic coefficient. This renders the theoretical bolometric amplitude closer to the observations. We are, however, left with a significant remaining difference.

\section{THREE DIMENSIONAL HYDRODYNAMICAL CONSTRAINTS ON MODE EIGENFUNCIONS}

In order to quantify the departure from adiabatic eigenfunction, we have derived at different layers the velocity profiles $v^{3 D}(r)$ of the dominant radial modes trapped in given 3D hydrodynamical models of the surface layer of stars. We consider two 3D hydrodynamical models. The first one is representative for a red giant star with $\log g=2.5$ and $T_{\text {eff }}=4964 \pm 22 \mathrm{~K}$ (see details in [29]), and was computed with $\mathrm{CO}^{5} \mathrm{BOLD}$ code [30]. The second one is representative for the Sun [31] and was computed with the STAGGER code $[32,33]$. Both 3D models have been run during a sufficient duration such as to resolve the dominant trapped radial modes. The procedure followed for the derivation of $v_{3 \mathrm{D}}(r)$ will be detailed in a forthcoming paper.

We compare $v_{3 \mathrm{D}}(r)$ with $v_{\mathrm{ad}}(r)$, the velocity profile associated with a radial adiabatic eigendisplacement, $\xi_{\mathrm{r}}$. The eigen-displacements, $\xi_{\mathrm{r}}$, were computed using the adiabatic pulsation code ADIPLS [34] and the global 1D "patched" models associated with the 3D models. Each patched model 

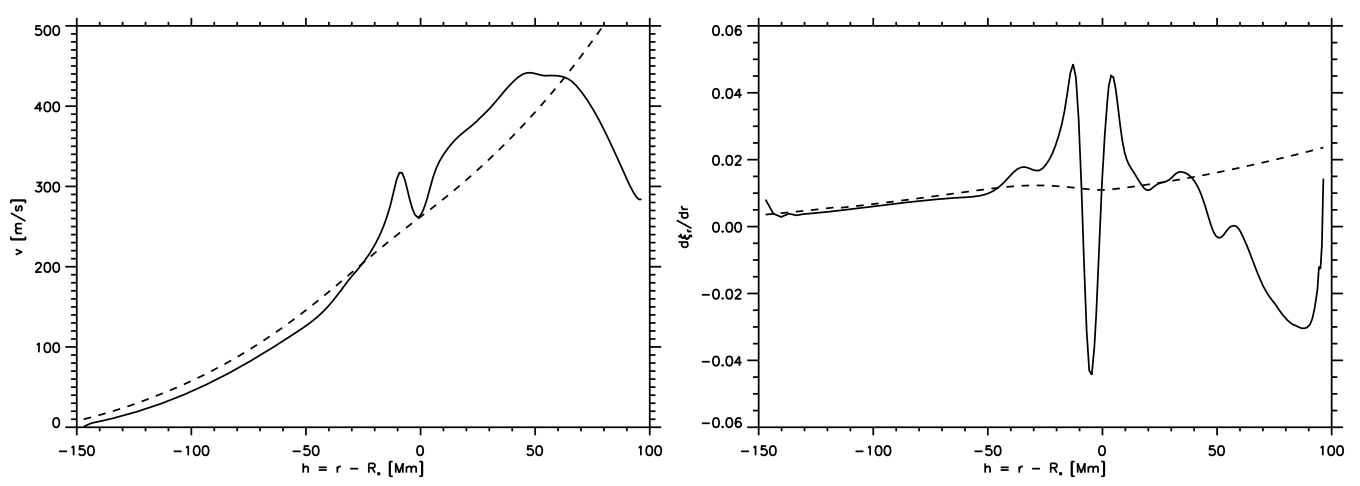

Figure 3. Left: Mode amplitude in velocity as a function of $h=r-R_{*}$. The solid line corresponds to $v_{3 \mathrm{D}}(r)$ the velocity profile of the dominant radial mode trapped in the red giant $3 \mathrm{D}$ model (this mode has a frequency $v=33.16 \mu \mathrm{Hz})$. The dashed line corresponds to $v_{\mathrm{ad}}(r)$ the velocity profile of the associated adiabatic eigenfunction $\left(v_{\mathrm{ad}}=31.4 \mu \mathrm{Hz}, n=8\right)$. This adiabatic eigenfunction is normalized such that its kinetic energy equals that of the dominant radial mode of the red giant 3D model. Right: Spatial derivative of the mode displacement, $\left(d \xi_{\mathrm{r}} / d r\right)$, as a function of $h$. The lines have the same meaning as in the left panel.

was computed in such a way that the outer layers are obtained from the 3D model [19] while the interior layers were computed using the CESAM2K code [35]. We normalized $\xi_{\mathrm{r}}$ such that the mode kinetic energy equals that of the trapped mode. Finally, we get $v_{\mathrm{ad}} \equiv 2 \pi v_{\mathrm{ad}} \xi_{\mathrm{r}}$, where $v_{\mathrm{ad}}$ is the adiabatic mode eigenfrequency. We choose the adiabatic mode that is both close in frequency and with a node close to that of the trapped mode.

\subsection{A representative red giant 3D model}

The dominant radial mode trapped in the red giant 3D model has a frequency $v=33.16 \mu \mathrm{Hz}$. Its velocity profile is plotted in Fig. 3 (left) as a function of $h=r-R_{*}$, where $R_{*}$ is the radius of the associated patched model. $v_{3 \mathrm{D}}(r)$ is compared with the velocity profile of the adiabatic mode that has a frequency $\nu_{\mathrm{ad}}=31.4 \mu \mathrm{Hz}$ and a radial order $n=8$. If we exclude the upper part of the atmosphere $(h \gtrsim 50 \mathrm{Mm})$ where the driving is anyway negligible because of the low density and much weaker convective motions, the difference between $v_{3 \mathrm{D}}(r)$ and $v_{\mathrm{ad}}(r)$ is the highest near the peak of the super-adiabatic gradient $(h \simeq-10 \mathrm{Mm})$.

At a given layer, the power injected into a given mode by the Reynolds stress depends on the mode compressibility, that is the term $\left(d \xi_{\mathrm{r}} / d r\right)$ in Eq. (4). The higher the mode compressibility, the stronger the driving by the Reynolds stress. The mode compressibility is related to the velocity as $\left(d \xi_{\mathrm{r}} / d r\right)=(2 \pi v)^{-1}(d v / d r)$. We have plotted in Fig. 3 (right) the spatial derivative of the displacement of the dominant mode (hereafter $\left.\left(d \xi_{\mathrm{r}} / d r\right)_{3 \mathrm{D}}\right)$ and that of the associated adiabatic mode (hereafter $\left.\left(d \xi_{\mathrm{r}} / d r\right)_{\mathrm{ad}}\right)$. If we ignore again the upper part of the atmosphere, the stronger variations of the velocity profile occur in the super-adiabatic layers between $h \simeq-50 \mathrm{Mm}$ and $h \simeq 0$.

$\left|\left(d \xi_{\mathrm{r}} / d r\right)_{3 \mathrm{D}}\right|$ reaches much higher values than $\left|(d \delta r / d r)_{\text {ad }}\right|$. As a consequence, use of a non-adiabatic mode must result in a stronger driving by the Reynold stress (the term $C_{\mathrm{R}}^{2}$ in Eq. (4)). In order to quantify this effect, we have computed for the mode with frequency $\nu_{\mathrm{ad}}=31.4 \mu \mathrm{Hz}$ the contribution of the Reynold stress using $\left(d \xi_{\mathrm{r}} / d r\right)_{3 \mathrm{D}}$ instead of $\left(d \xi_{\mathrm{r}} / d r\right)_{\mathrm{ad}}$. The mode excitation rate of this mode is found about three times larger than when the adiabatic eigenfuntion is used. In terms of amplitude, this corresponds to an amplitude $70 \%$ larger. This would put the theoretical mode amplitudes at the same level as the CoRoT measurements.

We turn now to the entropy source term (the term $C_{\mathrm{S}}^{2}$ in Eq. (5)). This term involves not only the first spatial derivative of the mode displacement but also the second derivative, $\left(d^{2} \xi_{\mathrm{r}} / d r^{2}\right)$. 


\section{Ageing Low Mass Stars: From Red Giants to White Dwarfs}
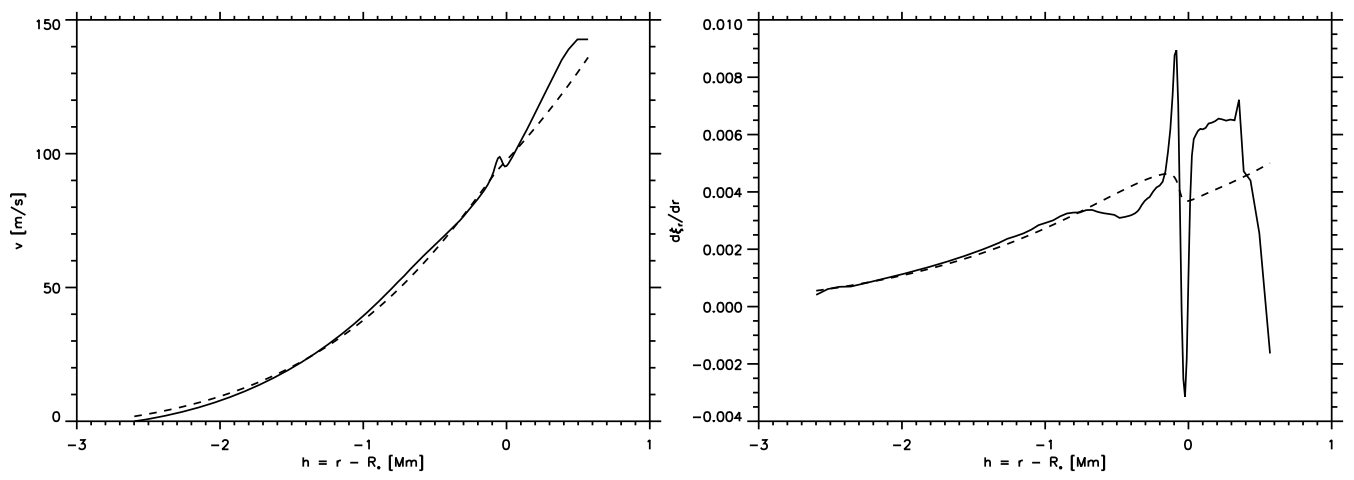

Figure 4. As for Fig. 3 for the case of a 3D model of the surface layers of the Sun. The lines have the same meaning as in Fig. 3. The dominant radial mode (solid line) trapped inside the solar 3D model has a frequency $v=2.607 \mathrm{mHz}$. The adiabatic eigenfunction (dashed line) has a frequency $v_{\text {ad }}=2.495 \mathrm{mHz}$ and a radial order $n=17$. Left: Mode amplitude in velocity. Right: Derivative of the mode displacement $\left(d \xi_{\mathrm{r}} / d r\right)$.

Departure of $\left(d^{2} \xi_{\mathrm{r}} / d r^{2}\right)$ from the adiabatic case is obviously expected to be stronger than for the first derivative $\left(d \xi_{\mathrm{r}} / d r\right)$. In the same way than done for the contribution of the Reynolds stress, we have quantified the impact of this departure in terms of mode excitation rate. We find that the driving due to the entropy source term is about 200 times larger than when the adiabatic eigenfuntion is assumed. In terms of amplitudes, this corresponds to an amplitude about 15 times larger. This would this time results in a severe over-estimation of the amplitudes. This result, must, however, be considered with caution (see the discussion in Sect. 5).

\subsection{Solar case}

Given the results obtained for a red giant 3D model (Sect. 4.1), we naturally reconsidered the previous theoretical calculations done for the case of the Sun and other stars. The question is then by which amount the theoretical mode excitation rates change for the Sun or other main-sequence stars if we use a non-adiabatic mode displacement derived from a 3D simulation instead of an adiabatic one.

To address this question, we have extracted in the same manner than done for the red giant model the velocity profile $v_{3 \mathrm{D}}(r)$ of the dominant radial mode trapped in the solar 3D model (this mode has a frequency $v=2.607 \mathrm{mHz}$ ). The result is shown in Fig. 4 (left) and compared with the velocity profile of the closest adiabatic mode $v_{\text {ad }}(r)$ computed for the patched 1D model associated with the solar 3D model. This adiabatic mode has a frequency $v_{\mathrm{ad}}=2.495 \mathrm{mHz}$ and a radial order $n=17$. The differences between $v_{3 \mathrm{D}}(r)$ and $v_{\mathrm{ad}}(r)$ are rather small except only near the peak of the super-adiabatic gradient ( $h \simeq-0.08 \mathrm{Mm}$, we again do not consider the upper part of the atmosphere, i.e. $h \gtrsim 0.1 \mathrm{Mm}$ ). These differences are qualitatively consistent with these found by [36]. However, again that matters for the driving are the first and second spatial derivatives of the mode displacement. We show in Fig. 4 (right), a comparison between $(d \delta r / d r)_{3 \mathrm{D}}$ and $(d \delta r / d r)_{\mathrm{ad}}$. As expected, the stronger differences occur near the peak of the super-adiabatic gradient.

We have estimated the amount by which theoretical mode excitation rates $\mathcal{P}$ are changed when we use the non-adiabatic mode obtained from the solar 3D model instead of the adiabatic one. At the frequency $v_{\mathrm{ad}}=2.495 \mathrm{mHz}$ (i.e. the frequency of the adiabatic mode close in frequency with the dominant mode of the solar 3D model), the driving by the Reynold stress is increased by $\sim 15 \%$. This increase remains smaller than the uncertainties associated with the current helioseismic data $[1,37]$. However, this amount is expected to be larger at higher frequency. Furthermore and more problematic, the entropy contribution is increased by a factor about 20. As for the red giant 3D model, this implies a severe overestimation of the solar mode amplitude (but see the discussion in Sect. 5). 


\section{EPJ Web of Conferences}

\section{DISCUSSION}

Large differences were found between the non-adiabatic radial mode trapped in a red giant 3D model and an equivalent adiabatic mode. Although less important, similar differences were found for a solar 3D model.

Before discussing the implications of these results in terms of mode amplitudes, we must first point out that these preliminary results must be considered with caution. Indeed, its is important to note that the trapped modes have much higher velocity than the solar-like oscillations. For instance, the solar oscillations can be as high as $30 \mathrm{~cm} / \mathrm{s}$ whereas the dominant radial mode of our solar 3D model reaches $150 \mathrm{~m} / \mathrm{s}$ at the atmosphere. This is obviously because the trapped modes have a much lower inertia (or equivalently mode mass, see Eq. (1) and Eq. (2)). For propagating acoustic wave, the relative density variation $\delta \rho / \rho$ is related to the wave velocity as $\delta \rho / \rho=v / c_{s}$. Accordingly, this implies a the photosphere $\delta \rho / \rho \sim 0.02$ for the mode trapped in the solar 3D model, and $\delta \rho / \rho \sim 0.07$ for the one trapped in the red giant 3D model. Therefore, the trapped modes are more away from the linear regime than are the global modes. For this reason, we must question their representativeness.

Furthermore, the first and second derivatives of the trapped mode displacement were computed numerically. The 3D models have a coarse vertical grid: the vertical resolution is about $1 \mathrm{Mm}$ and $15 \mathrm{~km}$ for the red giant model and the solar 3D model, respectively. Therefore, we have to check that the resolution is enough for a reliable calculation of the spatial derivatives, in particular for the second derivative, which is required for the calculation of the entropy source term. This said, we discuss below the implications of our results, but keeping in mind that a closer look to our results is required (work in progress).

The trapped non-adiabiatic radial modes were found significantly more compressible than the adiabatic ones. The higher the mode compressibility, the stronger driving. As a consequence, use of a non-adiabatic mode must result in a stronger driving. For the Reynolds stress contribution, use of the non-adiabatic trapped in the 3D model would result in a power $\mathcal{P}$ about three times larger. If we consider only the driving by the Renolds stress, the increase in the mode amplitude due to the departure from the adiabatic case would be of about $70 \%$ for representative red giant, leading predictions amplitudes very close to the seismic measurements done for red giants. For the entropy contribution (the thermal source of driving), the increase in amplitude would be by a factor about 15 . This would put the predicted mode amplitudes for red giant very far above the seismic measurements. If our results are confirmed, this would indicate a severe deficiency in the modelling of this thermal source of driving. We foresee two reasons that could explain such a deficiency:

- The theoretical model of stochastic excitation is based on the assumption that entropy fluctuations behave as a passive scalar [1]. This is a strong hypothesis that is unlikely to be valid in the superadiabatic part of the convective zone where both driving by the entropy is important and variations of the mode displacement are important. This assumption implies vanishing cross terms between the entropy fluctuations and the Reynolds stresses [26]. However, stellar 3D simulations performed by [38] show some partial canceling between the Reynolds stress contribution and non-adiabatic gas pressure fluctuations (which are directly related to entropy fluctuations). As originally suggested by [39] and discussed in [20], the existence of a partial canceling can decrease the mode amplitude and improve the agreement with the seismic observations. This partial cancelling can partially compensate the increase of the entropy contribution by the use of the non-adiabatic eigenfunction.

- All models of stochastic excitation by turbulent convection explicitly or implicitly assume that eddies, which effectively contribute to the driving, have a characteristic length scale smaller than the mode wavelength. In other words, it is assumed that the mode eigen-displacement varies at length scale significantly longer than the eddies contributing effectively to the driving. This assumption then permits to decouple spatially the the eigen-displacement from the source functions. As seen above the non-adiabatic mode displacement $\xi_{\mathrm{r}, 3 \mathrm{D}}$ derived from the $3 \mathrm{D}$ simulation varies spatially much 


\section{Ageing Low Mass Stars: From Red Giants to White Dwarfs}

rapidly than the adiabatic eigen-displacement $\xi_{\mathrm{r}, \mathrm{ad}}$ does. Therefore, assuming that the mode eigendisplacement is decoupled from the two driving sources (Reynolds stress and entropy fluctuations) is not valid in particular for the entropy source term, which is very localised near the super-adiabatic region.

The CoRoT space mission, launched on December 27 2006, has been developed and is operated by CNES, with the contribution of Austria, Belgium, Brasil, ESA, Germany and Spain.

\section{References}

[1] R. Samadi, Stochastic excitation of acoustic modes in stars, in The Pulsations of the Sun and the Stars, edited by J.P. Rozelot, C. Neiner (2011), Vol. 832 of Lecture Notes in Physics, Berlin Springer Verlag, arXiv:astro-ph/0912.0817, http://dx.doi.org/10.1007/9783-642-19928-8

[2] K. Belkacem, R. Samadi, Connections between stellar oscillations and turbulent convection (Springer Verlag, 2013), Vol. in press of Lecture Notes in Physics, Berlin Springer Verlag

[3] J. Christensen-Dalsgaard, S. Frandsen, Solar Physics 82, 469 (1983)

[4] H. Kjeldsen, T.R. Bedding, A\&A 293, 87 (1995)

[5] K. Belkacem, R. Samadi, M.J. Goupil, M.A. Dupret, A.S. Brun, F. Baudin, A\&A 494, 191 (2009), 0810.0602

[6] G. Houdek, N.J. Balmforth, J. Christensen-Dalsgaard, D.O. Gough, A\&A 351, 582 (1999)

[7] R. Samadi, D. Georgobiani, R. Trampedach, M.J. Goupil, R.F. Stein, Å. Nordlund, A\&A 463, 297 (2007), arXiv: astro-ph/0611762

[8] R. Samadi, K. Belkacem, M.A. Dupret, H.G. Ludwig, F. Baudin, E. Caffau, M.J. Goupil, C. Barban, A\&A 543, A120 (2012), 1205.4846

[9] E. Michel, R. Samadi, F. Baudin, C. Barban, T. Appourchaux, M. Auvergne, A\&A 495, 979 (2009), 0809.1078

[10] J. Ballot, C. Barban, C. van’t Veer-Menneret, A\&A 531, A124 (2011), 1105.4557

[11] F. Baudin, C. Barban, K. Belkacem, S. Hekker, T. Morel, R. Samadi, O. Benomar, M.J. Goupil, F. Carrier, J. Ballot et al., A\&A 529, A84 (2011), 1102.1896

[12] A. Grigahcène, M.A. Dupret, M. Gabriel, R. Garrido, R. Scuflaire, A\&A 434, 1055 (2005)

[13] R. Samadi, Å. Nordlund, R.F. Stein, M.J. Goupil, I. Roxburgh, A\&A 403, 303 (2003)

[14] R. Samadi, Å. Nordlund, R.F. Stein, M.J. Goupil, I. Roxburgh, A\&A 404, 1129 (2003)

[15] W.J. Chaplin, G. Houdek, Y. Elsworth, D.O. Gough, G.R. Isaak, R. New, MNRAS 360, 859 (2005)

[16] K. Belkacem, R. Samadi, M.J. Goupil, F. Kupka, F. Baudin, A\&A 460, 183 (2006), arXiv:astro-ph/0607570

[17] R. Samadi, K. Belkacem, M.J. Goupil, H.G. Ludwig, M.A. Dupret, Communications in Asteroseismology 157, 130 (2008)

[18] G. Houdek, D.O. Gough, MNRAS 336, L65 (2002)

[19] R. Samadi, K. Belkacem, M.J. Goupil, M.A. Dupret, F. Kupka, A\&A 489, 291 (2008), 0806.0725

[20] R. Samadi, H.G. Ludwig, K. Belkacem, M.J. Goupil, O. Benomar, B. Mosser, M.A. Dupret, F. Baudin, T. Appourchaux, E. Michel, A\&A 509, A16 (2010), 0910.4037

[21] N.J. Balmforth, MNRAS 255, 639 (1992)

[22] R. Samadi, M.J. Goupil, G. Houdek, A\&A 395, 563 (2002)

[23] N.J. Balmforth, MNRAS 255, 603 (1992)

[24] A.Z. Dolginov, A.G. Muslimov, Ap\&SS 98, 15 (1984) 
[25] P. Goldreich, N. Murray, P. Kumar, ApJ 424, 466 (1994)

[26] R. Samadi, M.J. Goupil, A\&A 370, 136 (2001)

[27] K. Belkacem, R. Samadi, M.J. Goupil, M.A. Dupret, A\&A 478, 163 (2008), arXiv: 0710.1039

[28] P. Goldreich, D.A. Keeley, ApJ 212, 243 (1977)

[29] H.G. Ludwig, M. Steffen, ArXiv e-prints (2011), 1108.4554

[30] B. Freytag, M. Steffen, H.G. Ludwig, S. Wedemeyer-Böhm, W. Schaffenberger, O. Steiner, Journal of Computational Physics 231, 919 (2012), 1110.6844

[31] K. Belkacem, R. Samadi, M.J. Goupil, F. Kupka, A\&A 460, 173 (2006), arXiv: astro-ph/0607568

[32] R.F. Stein, A. Nordlund, ApJ 499, 914 (1998)

[33] R. Trampedach, Master's thesis, Master's thesis, Aarhus University (1997) (1997)

[34] J. Christensen-Dalsgaard, Ap\&SS 316, 113 (2008), 0710.3106

[35] P. Morel, Y. Lebreton, Ap\&SS 316, 61 (2008), 0801.2019

[36] R.F. Stein, Å. Nordlund, ApJ 546, 585 (2001)

[37] F. Baudin, R. Samadi, M.J. Goupil, T. Appourchaux, C. Barban, P. Boumier, W.J. Chaplin, P. Gouttebroze, A\&A 433, 349 (2005)

[38] R. Stein, D. Georgobiani, R. Trampedach, H.G. Ludwig, Å. Nordlund, Solar Physics 220, 229 (2004)

[39] G. Houdek, Stochastic excitation and damping of solar-like oscillations, in Proceedings of SOHO 18/GONG 2006/HELAS I, Beyond the spherical Sun (2006), Vol. 624 of ESA Special Publication, pp. Published on CDROM, p. 28.1 